\title{
Systematic errors in partially-quenched QCD plus QED lattice simulations
}

\author{
A. Portelli ${ }^{* b}$, S. Dürr ${ }^{a, d}$, Z. Fodor ${ }^{a, c, d}$, J. Frison ${ }^{b}$, C. Hoelbling ${ }^{a}$, S.D. Katz ${ }^{a, c}$, \\ S. Krieg ${ }^{a, d}$, T. Kurth ${ }^{a}$, L. Lellouch ${ }^{b}$, T. Lippert ${ }^{a}$, A. Ramos ${ }^{b}$ and K.K. Szabó ${ }^{a}$ \\ (Budapest-Marseille-Wuppertal Collaboration) \\ ${ }^{a}$ Bergische Universität Wuppertal, Gaußstr. 20, D-42097 Wuppertal, Germany \\ ${ }^{b}$ Centre de Physique Théorique, Aix-Marseille Univ, CNRS UMR 7332, Univ Sud Toulon Var, \\ 13288 Marseille cedex 9, France ${ }^{\dagger}$ \\ ${ }^{c}$ Institute for Theoretical Physics, Eötvös University, H-1117 Budapest, Hungary \\ ${ }^{d}$ Jülich Supercomputing Centre, Forschungszentrum Jülich, D-52425 Jülich, Germany \\ E-mail : portelli@cpt.univ-mrs.fr
}

\begin{abstract}
At the precision reached in current lattice QCD calculations, electromagnetic effects are becoming numerically relevant. Here, electromagnetic effects are included by superimposing U(1) degrees of freedom on $N_{f}=2+1$ QCD configurations from the Budapest-Marseille-Wuppertal Collaboration. We present preliminary results for the electromagnetic corrections to light pseudoscalars mesons masses and discuss some of the associated systematic errors.
\end{abstract}

The XXIX International Symposium on Lattice Field Theory - Lattice 2011

July 10-16, 2011

Squaw Valley, Lake Tahoe, California

\footnotetext{
* Speaker.

†Unité Mixte de Recherche (UMR 7332) du CNRS, de l'Université d'Aix-Marseille et de l'Université Sud Toulon Var. Unité affiliée à la FRUMAM, Fédération de Recherche 2291 du CNRS.
} 


\section{Motivation}

Isospin is a near symmetry of the hadron spectrum because it is only broken by small effects:

(i) the mass difference $m_{u}-m_{d}$

(ii) the difference in the charge of the $u$ and the $d$ quark

which are summarized in the following table ${ }^{1}$ :

\begin{tabular}{|l|c|c|}
\cline { 2 - 3 } \multicolumn{1}{c|}{} & $u$ & $d$ \\
\hline Mass $(\mathrm{MeV})[1,2]$ & $2.15(03)_{\text {stat. }}(10)_{\text {sys. }}$ & $4.79(07)_{\text {stat. }}(12)_{\text {sys. }}$ \\
Charge & $\frac{2}{3} e$ & $-\frac{1}{3} e$ \\
\hline
\end{tabular}

These effects are expected to be at the percent level. The size of mass breaking is the mass difference $m_{u}-m_{d}$ relatively to a typical QCD scale $\Lambda_{\mathrm{QCD}}$ and the order of electromagnetic breaking is the fine structure constant at zero momentum $\alpha=\frac{e^{2}}{4 \pi} \simeq \frac{1}{137}$. However, their importance is not commensurate to their size. For instance, they are responsible for the stability of matter through the proton-neutron mass difference. Moreover, lattice QCD calculations have recently approached percent or even sub-percent precision [4,5], so the inclusion of these effects becomes relevant.

Another interesting isospin breaking quantity is the absolute correction to Dashen's theorem

$$
\Delta_{A} D=\Delta_{\mathrm{EM}} M_{K}^{2}-\Delta_{\mathrm{EM}} M_{\pi}^{2}
$$

where :

$$
\Delta_{\mathrm{EM}} M_{P}^{2}=\left(M_{P^{+}}^{2}-M_{P^{0}}^{2}\right)_{m_{u}=m_{d}}
$$

is the electromagnetic squared mass splitting of the isospin multiplet $P$. One can also consider the dimensionless relative correction to Dashen's theorem:

$$
\Delta_{R} D=\frac{\Delta_{\mathrm{EM}} M_{K}^{2}}{\Delta_{\mathrm{EM}} M_{\pi}^{2}}-1
$$

R. Dashen has shown in [6] than $\Delta_{A} D=0$ in the SU(3) chiral limit and that the leading corrections are $\mathrm{O}\left(\alpha m_{s}, \alpha^{2}\right)$. The quantity $\Delta_{A} D$ is interesting because it is very sensitive to the up and down quark masses. Moreover, a precise estimation of these quantities has not been made until now (cf. Table 1).

\section{Simulation setup}

For this work, we used a subset of our $2010 \mathrm{SU}$ (3) gauge configurations [2]. These configurations were generated using $N_{f}=2+1$ QCD simulations with the tree-level Symanzik gauge action, tree level $\mathrm{O}(a)$-improved Wilson fermions and two steps of HEX smearing. We have already used this dataset to compute light quark masses [1,2] and the kaon bag parameter [18].

\footnotetext{
${ }^{1}$ We have chosen to quote the quark masses from $[1,2]$. Please see [3] for a complete list of lattice determinations of these masses.
} 


\begin{tabular}{|c|c|c|c|}
\hline & $\Delta_{A} D\left(\mathrm{MeV}^{2}\right)$ & $\Delta_{R} D$ & \\
\hline \multirow{6}{*}{ 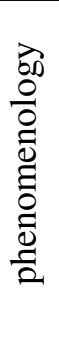 } & 1230 & 0.80 & Donoghue et al. [7] (1993) \\
\hline & $1300(400)$ & $1.0(3)$ & Bijnens [8] (1993) \\
\hline & 360 & 0.26 & Baur and Urech [9] (1996) \\
\hline & $1060(320)$ & $0.9(4)$ & Bijnens and Prades [10] (1997) \\
\hline & 1080 & 0.68 & Gao et al. [11] (1997) \\
\hline & 1070 & 0.74 & Bijnens and Danielsson [12] (2007) \\
\hline \multirow{5}{*}{ 总 } & 526 & 0.39 & Duncan et al. [13] (1996) \\
\hline & $340(92)$ & $0.30(8)$ & Blum et al. [14] (2007) \\
\hline & $1250(550)$ & N/A & Basak et al. [15] (2008) \\
\hline & $830(180)$ & $0.60(14)$ & Portelli et al. [16] (2011) \\
\hline & 707(75) & $0.63(6)$ & Blum et al. [17] (2010) \\
\hline
\end{tabular}

Table 1: Results for the violations to Dashen's theorem in phenomenelogy and lattice computations. Order is chronological. Bold numbers are the results given by the authors, the others are deduced from information given in the corresponding paper.

To include QED, we generate real electromagnetic fields $A_{\mu}$ using a non-compact formulation ( $c f$. [16] for more details). Then we phase $\mathrm{SU}(3)$ strong links by the $\mathrm{U}(1)$ links $\exp \left(i Q A_{\mu}\right)$, where $Q$ is a chosen electric charge. The resulting $\mathrm{U}(3)$ links are used inside the Dirac-Wilson operator to compute quark propagators. This method allows to use previously generated $\mathrm{SU}(3)$ gauge configurations to obtain results, but the electromagnetic vacuum polarization is not taken into account. For the preliminary study presented here, the masses of the up and down valence quarks are tuned individually such that the bare masses are equal. This occurs when these masses become equal to the light sea quark mass [16]. The $\pi^{0}$ squared mass is obtained by averaging squared ground state energies obtained with $\bar{u} u$ and $\bar{d} d$ connected pseudoscalar correlators. This is correct up to NLO isospin breaking corrections.

In this setup, one is confronted with two new types of systematics effects : the quenching of the electromagnetic field and electromagnetic finite volume effects.

\section{QED quenching errors}

Using photon fields without vaccuum polarization leads to partial quenching effects: valence quarks have electric charges but sea quarks do not. These effects can be evaluated in partially quenched chiral perturbation theory with QED (PQ $\chi$ PT+QED). Next to leading order (NLO) $\mathrm{SU}(3)$ formulas for self-energies and decay constants can be found in $[12,17]$. The SU(3) NLO sea contribution to a pseudoscalar meson squared mass is given by :

$$
\delta_{\text {sea }} M_{i j}^{2}=\frac{e^{2} C}{8 \pi^{2} F_{0}^{4}}\left(q_{i}-q_{j}\right) \sum_{s=4}^{6} q_{s}\left[\chi_{i s} \log \left(\frac{\chi_{i s}}{\mu}\right)-\chi_{j s} \log \left(\frac{\chi_{j s}}{\mu}\right)\right]-\frac{e^{2}}{3} Y_{1} \chi_{i j} \sum_{s=4}^{6} q_{s}^{2}
$$

where $i$ and $j$ are the valence indices of the quarks composing the meson, indices, $s$, between 4 and 6 are sea quark indices, $\chi_{k l}=B_{0}\left(m_{k}+m_{l}\right), e \simeq 0.302822$ is the positron electric charge, $q_{k}$ are quark charges in units of $e$ and $Y_{1}, C$ and $F_{0}$ are low energy constants (LECs). 
For the results presented here, the sea and valence masses are very nearly equal and we can assume that the absence of sea charges is the only partial quenching effect. In that case, one can check easily that (3.1) is independant of the scale $\mu$. The LECs $C$ and $F_{0}$ are essentially known [12], but $Y_{1}$, which is a sea electromagnetic contribution, is unknown. RBC, who studied other partiallyquenched QED LECs [14, 17], considers that it would be unnatural that $Y_{1}>10^{-2}$. Thus, in the following, we will use $Y_{1}=10^{-2}$. With (3.1), one obtains the following partial quenching error estimates:

- negligible (less than $0.1 \%$ ) for $M_{K^{+}}, M_{K^{0}}, \Delta_{\mathrm{EM}} M_{\pi}$ and $\Delta_{\mathrm{EM}} M_{\pi}^{2}$

- $\sim 0.4 \%$ for $M_{\pi^{0}}$

- $\sim 5 \%$ for $\Delta_{\mathrm{EM}} M_{K}$ and $\Delta_{\mathrm{EM}} M_{K}^{2}$

\section{Electromagnetic finite volume effects}

It is already known [2, p. 18] that the Budapest-Marseille-Wuppertal gauge ensembles have spatial volumes that allow to neglect QCD finite volume effects. However, as electromagnetism is a long range interaction, one might expect large QED finite volume effects on a periodic lattice. Predictions for these effects have been made in PQ $\chi$ PT+QED $[19,17]$. These formulas are far from simple and complicate chiral fits. Additionally, their predictivity seems limited [17, p. 16].

Here we choose a more straightforward approach. Dimensional analysis suggests that the leading finite volume correction to the splitting of a squared hadron mass, $\Delta M_{h}^{2}$, takes the form :

$$
\Delta M_{h}^{2}(\infty)-\Delta M_{h}^{2}(L)=e^{2} \frac{A}{L^{2}}
$$

where $L$ is the spatial extent of the lattice and $A$ is an unknown dimensionless constant. We found, as presented in the next section, that finite volume corrections of type (4.1) fit lattice data well but are not compatible with SU(3) PQ $\chi \mathrm{PT}+\mathrm{QED}$ ( $c f$. Figure 1).

\section{Preliminary results}

For this preliminary analysis, we used the $\beta=3.31$ subset of Budapest-Marseille-Wuppertal 2-HEX gauge configurations [2]. The main features of this dataset are:

- one lattice spacing $a \simeq 0.12 \mathrm{fm}$

- 12 pion masses from $135 \mathrm{MeV}$ to $422 \mathrm{MeV}$

- 4 spatial volumes from $(2 \mathrm{fm})^{3}$ to $(5.8 \mathrm{fm})^{3}$, with $M_{\pi^{0}} L>4$

To interpolate a quantity to the physical masses, we use a Taylor expansion in $M_{\pi^{0}}^{2}$ and $M_{K_{\chi}}^{2}=\frac{1}{2}\left(M_{K^{+}}^{2}+M_{K^{0}}^{2}-M_{\pi^{+}}^{2}\right)$. We choose $M_{\pi^{0}}^{2}$ because it has negligible finite volume corrections compared to those in $M_{\pi^{+}}^{2}$. The lattice spacing is computed in physical units using the $\Omega^{-}$baryon mass. The infinite volume extrapolation is performed by including corrections of the kind (4.1) into the fit. Fits are carried out using fully correlated $\chi^{2}$ minimization and a bootstrap error analysis. 


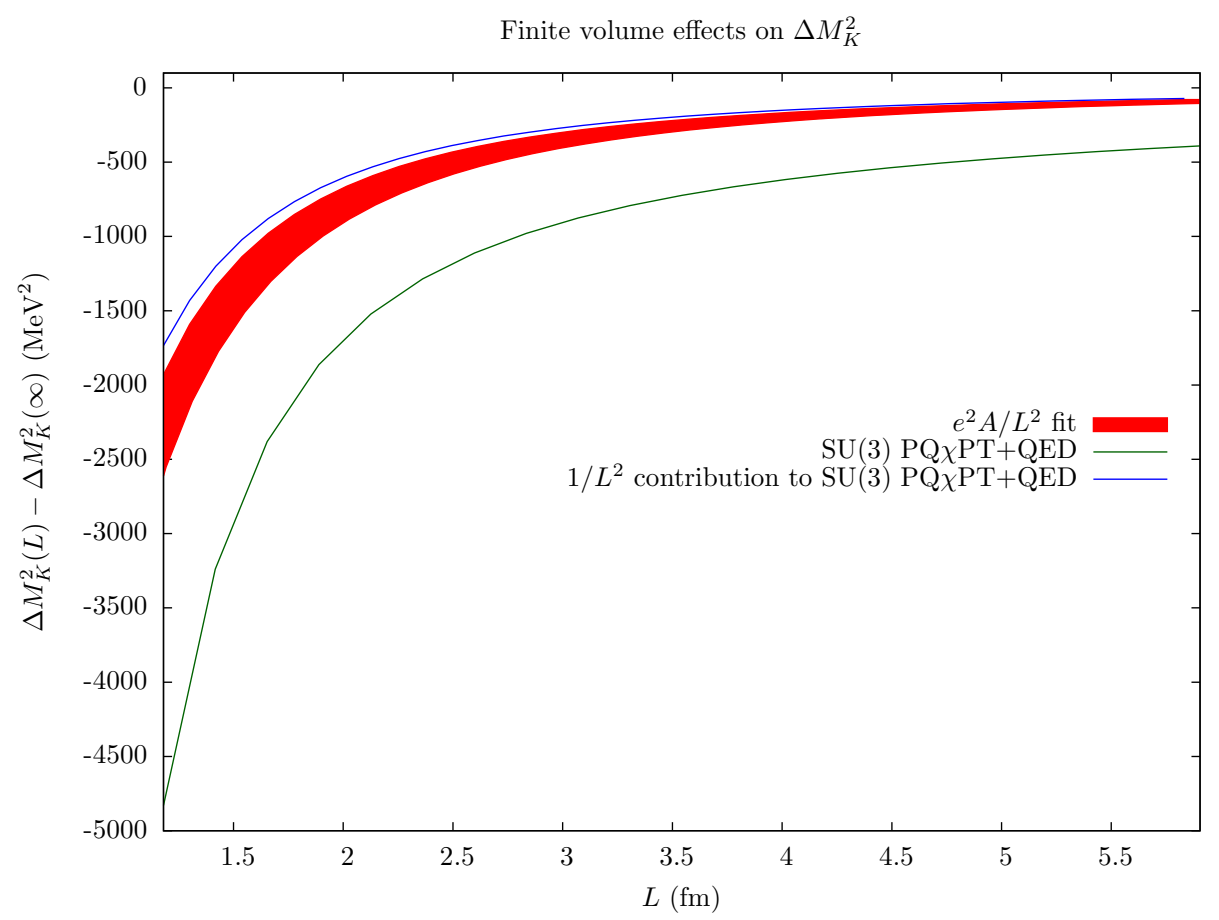

Figure 1: Comparison of our results with different models of finite volume corrections for the kaon squared mass splitting, as a function of lattice spatial extent. The red band represents the fit of our lattice data to the model (4.1), with $M_{\pi^{0}}$ fixed to its physical value. Only statistical errors are shown. The green curve represents the prediction of $\mathrm{SU}(3) \mathrm{PQ} \chi \mathrm{PT}+\mathrm{QED}$, which depends only on the LO, LECs $F_{0}$ and $C$, given in [12]. There is a clear disagreement between the two. However, the $\frac{1}{L^{2}}$ contribution from the full SU(3) $\mathrm{PQ} \chi \mathrm{PT}+\mathrm{QED}$ expression (blue curve) roughly agrees with our data.

Using this methodology, we obtain the following preliminary results (an example of such a fit is shown in Figure 2):

$$
\begin{aligned}
\Delta_{\mathrm{EM}} M_{K}^{2} & =2179(34)_{\text {stat. }}(100)_{\text {qu. }}(?)_{\text {sys. }} \mathrm{MeV}^{2} \\
\Delta_{\mathrm{EM}} M_{\pi}^{2} & =1283(30)_{\text {stat. }}(0)_{\text {qu. }}(?)_{\text {sys. }} \mathrm{MeV}^{2} \\
\Delta_{A} D & =896(37)_{\text {stat. }}(100)_{\text {qu. }}(?)_{\text {sys. }} \mathrm{MeV}^{2} \\
\Delta_{R} D & =0.70(4)_{\text {stat. }}(8)_{\text {qu. }}(?)_{\text {sys. }}
\end{aligned}
$$

where $(?)_{\text {sys. }}$ stands for the systematic errors which we have not yet estimated, such as those associated with taking the continuum limit, etc.

\section{Conclusion}

Using the methodology that we presented last year [16], we extended our dataset down to the physical value of the light quark mass. We also studied two important systematics effects: QED quenching and finite volume effects.

Our results are promising and close to phenomenological estimates [3]. Moreover, concerning the finite volume effects, the results are compatible with a simple $\frac{1}{L^{2}}$ model and it seems that $\mathrm{SU}(3)$ $\mathrm{PQ} \chi \mathrm{PT}+\mathrm{QED}$ fails to describe them. 
In the short term, we will continue our analysis on several lattice spacings with non-degenerate up and down quark masses. This will provide the last ingredients to define precisely the physical point, which require a continuum limit and physical isospin breaking.

\section{Acknowledgments}

Computations were performed using HPC resources from FZ Jülich and from GENCI-[IDRIS/CCRT] (grant 52275) and clusters at Wuppertal and CPT. This work is supported in part by EU grants I3HP, FP7/2007-2013/ERC No. 208740, MRTN-CT-2006-035482 (FLAVIAnet), DFG grants FO 502/2, SFB-TR 55, by CNRS grants GDR 2921 and PICS 4707.

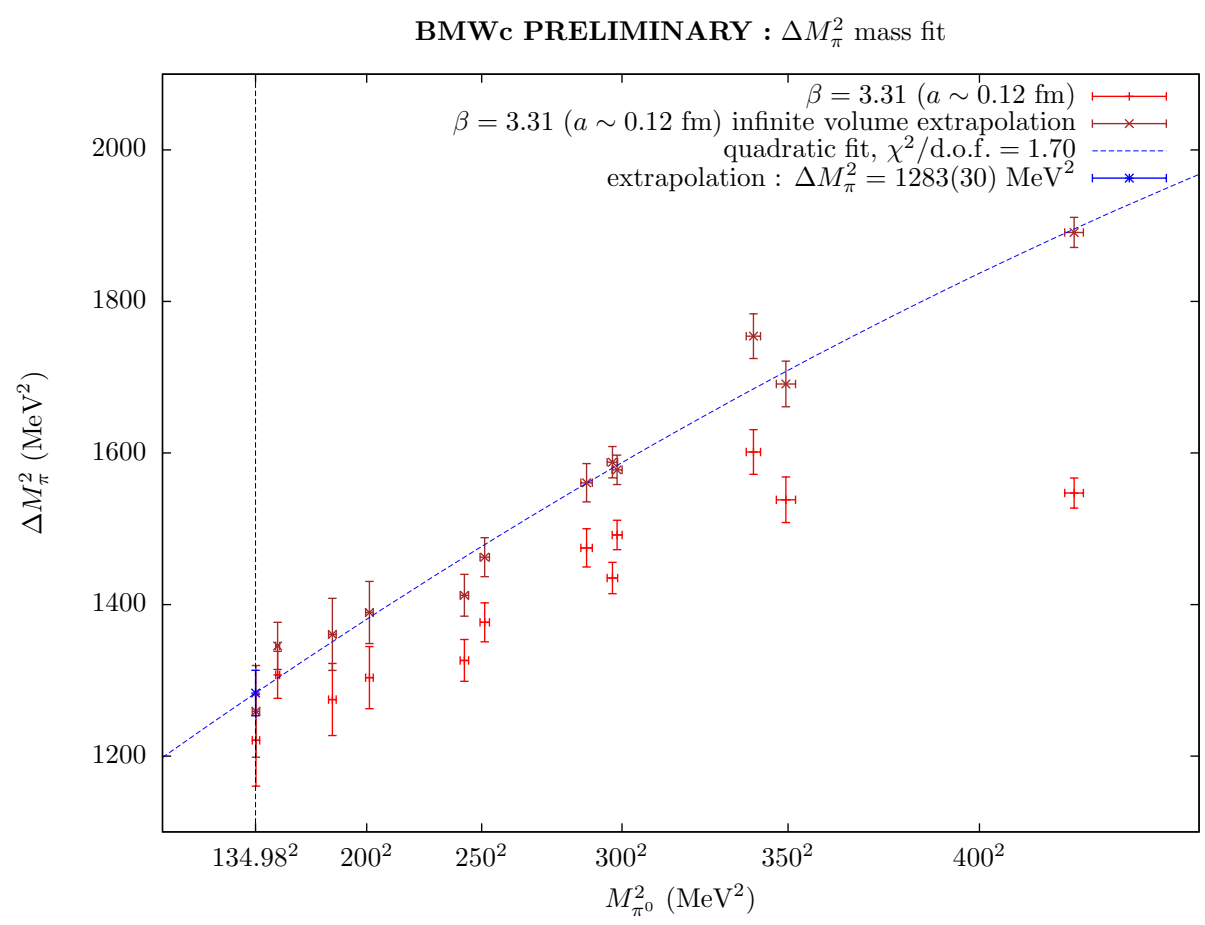

Figure 2: Pion squared mass splitting vs. neutral pion squared mass. Red points represent raw lattice data, dark red points are the same data with finite volume correction of type (4.1) and the dashed blue line is the result of the physical point fit.

\section{References}

[1] S. Dürr et al. Lattice QCD at the physical point: light quark masses. Physics Letters B 701(2) pp. 265-268 (2011)

[2] S. Dürr et al. Lattice QCD at the physical point: Simulation and analysis details. JHEP (8) pp. 1-47 (2011)

[3] G. Colangelo et al. Review of lattice results concerning low energy particle physics (2011). hep-lat/1011.4408 
[4] C. Hoelbling. Light hadron spectroscopy and pseudoscalar decay constants. PoS (Lattice 2010) (2011). hep-lat/1102.0410

[5] R. Mawhinney. Direct and Indirect Kaon Physics Directly Below KT-22: A Lattice 2011 Review. PoS (Lattice 2011) (2012). To be published

[6] R. Dashen. Chiral $\mathrm{SU}(3) \otimes \mathrm{SU}(3)$ as a symmetry of the strong interactions. Physical Review 183(5) pp. 1245-1260 (1969)

[7] J. F. Donoghue, B. R. Holstein and D. Wyler. Electromagnetic self-energies of pseudoscalar mesons and Dashen's theorem. Physical Review D 47(5) pp. 2089-2097 (1993)

[8] J. Bijnens. Violations of Dashen's theorem. Physics Letters B 306(3-4) pp. 343-349 (1993)

[9] R. Baur and R. Urech. Corrections to Dashen's theorem. Physical Review D 53(11) pp. 6552-6557 (1996)

[10] J. Bijnens and J. Prades. Electromagnetic corrections for pions and kaons: Masses and polarizabilities. Nuclear Physics B 490(1-2) pp. 239-271 (1997)

[11] D.-N. Gao, B. A. Li and M.-L. Yan. Electromagnetic mass splittings of $\pi, a_{1}, K, K_{1}(1400)$, and $K^{*}(892)$. Physical Review D 56(7) pp. 4115-4132 (1997)

[12] J. Bijnens and N. Danielsson. Electromagnetic corrections in partially quenched chiral perturbation theory. Physical Review D 75(1) p. 014505 (2007)

[13] A. Duncan, E. Eichten and H. Thacker. Electromagnetic splittings and light quark masses in lattice QCD. Physical Review Letters 76(21) pp. 3894-3897 (1996)

[14] T. Blum et al. Determination of light quark masses from the electromagnetic splitting of pseudoscalar meson masses computed with two flavors of domain wall fermions. Physical Review D 76(11) p. 114508 (2007)

[15] S. Basak et al. Electromagnetic splittings of hadrons from improved staggered quarks in full QCD. PoS (Lattice 2008) (2008). hep-lat/0812.4486

[16] A. Portelli et al. Electromagnetic corrections to light hadron masses. PoS (Lattice 2010) (2011). hep-lat/1011.4189

[17] T. Blum et al. Electromagnetic mass splittings of the low lying hadrons and quark masses from 2+1 flavor lattice QCD+QED. Physical Review D 82 p. 094508 (2010)

[18] S. Dürr et al. Precision computation of the kaon bag parameter. Physics Letters B 705(5) pp. 477-481 (2011)

[19] M. Hayakawa and S. Uno. QED in finite volume and finite size scaling effect on electromagnetic properties of hadrons (2008). hep-lat/0804.2044 